\section{Validade relativa de indicadores de práticas alimentares da Pesquisa Nacional de Saúde do Escolar entre adolescentes do Rio de Janeiro, Brasil}

\author{
Relative validity of dietary indicators from the \\ Brazilian National School-Based Health Survey \\ among adolescents in Rio de Janeiro, Brazil
}

\author{
Validez relativa de indicadores de prácticas \\ alimentarias en la Investigación Nacional de \\ Salud Escolar entre los adolescentes en \\ Río de Janeiro, Brasil
}

\author{
1 Instituto de Nutrição, \\ Universidade do Estado do \\ Rio de Janeiro, Rio de Janeiro, \\ Brasil. \\ 2 Faculdade de Medicina, \\ Universidade de São Paulo, \\ São Paulo, Brasil. \\ 3 Escola Nacional de Saúde \\ Pública Sergio Arouca, \\ Fundação Oswaldo Cruz, \\ Rio de Janeiro, Brasil. \\ Correspondência \\ L. F. Tavares \\ Instituto de Nutrição, \\ Universidade do Estado do \\ Rio de Janeiro. \\ Rua São Francisco Xavier \\ 524, 12 o andar, Bloco E, sala \\ 12.007, Rio de Janeiro, $R J$ \\ 20559-900, Brasil. \\ tavares.nutricao@gmail.com
}

\begin{abstract}
This study evaluated the relative validity of the dietary indicators from the questionnaire used in the Brazilian National School-Based Health Survey (PeNSE) in the city of Rio de Janeiro, Brazil. The sample included 174 students. The following indicators were evaluated: regular consumption ( $\geq 5$ times a week) of markers for healthy eating, markers for unhealthy eating, and routine eating habits (having meals with parents or guardians (MEAL), having breakfast (breakfast), and eating while studying or watching $T V$. The results of the questionnaire were compared with three 24-hour recalls. For all the markers of healthy eating, there was no difference in the proportion of regular consumption when comparing the two methods. The rates detected by the questionnaire were higher for packaged snacks and crackers and lower for cold cuts and MEAL. The indicators for regular consumption of markers for healthy eating and that referring to BREAKFAST were those with the highest accuracy. The dietary indicators used by the PeNSE survey showed satisfactory validity.
\end{abstract}

Food Consumption; Food Habits; Validation Studies; Adolescent
Letícia Ferreira Tavares 1

Inês Rugani Ribeiro de Castro 1

Renata Bertazzi Levy 2

Letícia Oliveira Cardoso 3

Michelle Delboni dos Passos 1

Flávia dos Santos Barbosa Brito ${ }^{1}$

\section{Resumo}

Avaliou-se a validade relativa dos indicadores de práticas alimentares do questionário utilizado na Pesquisa Nacional de Saúde do Escolar (PeNSE) entre adolescentes da cidade do Rio de Janeiro, Brasil. Foram estudados 174 alunos. Foram avaliados os indicadores: a ingestão regular ( $\geq 5$ vezes/semana) dos alimentos marcadores de alimentação saudável, marcadores de alimentação não saudável e as rotinas alimentares: realizar refeição com responsável (REFEIÇÃO), realizar o desjejum (DESJEJUM) e comer enquanto estuda ou assiste à TV. Foram comparados os resultados do questionário e dos três recordatórios de 24 horas. Para todos os marcadores de alimentação saudável, não houve diferença na proporção de ingestão regular de acordo com os dois métodos. As frequências geradas pelo questionário foram superiores para os indicadores salgadinhos de pacote e biscoitos salgados e mais baixas para embutidos e REFEIÇÃO. Os indicadores de consumo regular de marcadores de alimentação saudável e aquele referente a DESJEJUM foram os que apresentaram maior acurácia. Os indicadores de práticas alimentares utilizados pela PeNSE apresentaram validade satisfatória.

Consumo de Alimentos; Hábitos Alimentares; Estudos de Validação; Adolescente 


\section{Introdução}

Um componente fundamental para o norteamento e a avaliação de ações mais efetivas de prevenção e controle das doenças crônicas não transmissíveis (DCNT) é o conhecimento da magnitude, distribuição e tendência temporal dos fatores de risco para essas doenças nas populações para as quais essas políticas são dirigidas 1,2 . Nessa perspectiva, organismos internacionais recomendam que sejam desenvolvidas ações de monitoramento de fatores de risco e proteção à saúde associados às DCNT e destacam a importância de se desenvolverem sistemas de vigilância dirigidos aos adolescentes 3 .

Em consonância com essa recomendação e baseando-se em experiências de outros países 4,5,6 e brasileiras 2,7,8, a Secretaria de Vigilância em Saúde do Ministério da Saúde, em parceria com o Ministério da Educação e com o Instituto Brasileiro de Geografia e Estatística (IBGE), estruturou um sistema de vigilância de fatores de risco para a saúde de adolescentes baseado em inquéritos regulares realizados em escolas. $\mathrm{O}$ primeiro inquérito, denominado Pesquisa $\mathrm{Na}$ cional de Saúde Escolar (PeNSE), foi realizado, no ano de 2009, em uma amostra probabilística de adolescentes do 9o ano do Ensino Fundamental de escolas públicas e privadas das 26 capitais de estados brasileiros e do Distrito Federal 9. Nova edição desse inquérito ocorreu em 201210.

A precisão e possibilidade de inferência sobre estimativas geradas com base nos indicadores utilizados pelo sistema de vigilância dependem de avaliações da validade e confiabilidade do instrumento utilizado 11. Em âmbito internacional, os estudos de validação dos instrumentos utilizados pelos sistemas de vigilância dirigidos a adolescentes englobam módulos específicos como transtornos alimentares 12 , atividade física 13,14,15 e práticas alimentares 16,17,18.

Tendo em vista a ausência de estudos de validação do questionário utilizado pelo sistema de vigilância de fatores de risco para a saúde de adolescentes brasileiros, o presente estudo tem como objetivo analisar a validade relativa dos indicadores do módulo de práticas alimentares do questionário utilizado na PeNSE.

\section{Metodologia}

\section{População do estudo e amostragem}

A população de estudo foi composta por alunos que cursavam, em 2011, o 9o ano do Ensino Fundamental em escolas públicas e privadas da cidade do Rio de Janeiro. Os resultados obtidos em estudo de reprodutibilidade do questionário utilizado pelo sistema de monitoramento dessa cidade serviram de base para a definição do tamanho amostral do presente estudo 19 . A amostra foi dimensionada considerando-se o menor valor de kappa observado $(0,32$, para a questão referente à frequência de consumo de alimentos embutidos), a prevalência de consumo frequente (pelo menos cinco dias na semana) desse grupo de alimentos (20\%), precisão absoluta de 0,20 e nível de $95 \%$ de confiança, totalizando 126 alunos. O cálculo foi realizado implementando-se a rotina sskdlg (sample size for the kappa-statistic measure of interrater agreement) no programa Stata v. 10 (Stata Corp., College Station, Estados Unidos) 11,20 . Assumindo um percentual de perdas de $50 \%$ na segunda etapa do estudo (três entrevistas por meio de contato telefônico), trabalhamos com uma amostra inicial de 250 alunos.

Dados do Censo Escolar de 2010, conduzido pelo Ministério da Educação, foram utilizados como base para o sorteio e a seleção das turmas. Das 3.352 escolas existentes, 1.093 possuíam turmas de 9 o ano. Foram excluídas 152 turmas consideradas não elegíveis por apresentarem menos de 15 alunos. De acordo com o Censo Escolar de 2010, as turmas de escolas públicas e privadas possuíam, em média, 36,2 e 25,7 alunos, respectivamente, sendo necessário, portanto, a seleção de aproximadamente nove turmas para atingir o tamanho amostral. A distribuição do número de turmas por dependência administrativa da escola tomou, por base, a proporção de alunos matriculados em escolas públicas $(75 \%)$ e privadas (25\%) do município.

A seleção das turmas se deu por meio de amostragem sistemática, e o intervalo de seleção foi calculado de acordo com o número total de turmas do 9o ano das escolas públicas e privadas. Foram sorteadas nove turmas distribuídas em nove escolas (seis públicas e três privadas) espalhadas por toda a cidade do Rio de Janeiro.

\section{Questionário de práticas alimentares (método teste)}

Os assuntos contemplados no questionário da PeNSE são: características sociodemográficas, alimentação, imagem corporal, atividade física, tabagismo, consumo de álcool e outras drogas, saúde bucal, comportamento sexual, violência, acidentes, segurança, dados antropométricos (autorreferidos) e apreciação geral do questionário.

A ingestão alimentar foi medida por meio de questionário qualitativo (QUEST), no qual foi registrado o número de dias, na semana que antecedeu o estudo, em que o aluno consumiu: feijão; legume ou verdura, crua ou cozida, 
excluindo batata e aipim; salada crua; legume ou verdura cozidos na comida ou sopa (excluindo batata e aipim); frutas frescas ou salada de fruta; leite (exceto leite de soja); guloseimas (doces, balas, chicletes, pirulitos, chocolates ou bombons); biscoitos, bolachas, salgadinhos de pacote ou batata frita de pacote; biscoitos doces ou bolachas doces; biscoitos salgados ou bolachas salgadas; salgadinhos de pacote ou batata frita de pacote; batata frita (exceto a batata de pacote) ou salgado frito; embutidos (hambúrguer, salsicha, linguiça, mortadela, salame, presunto, peito de peru ou nuggets); refrigerante; bebidas com açúcar como refrigerantes, sucos ou refrescos, chás, águas com sabor, isotônicos, bebidas à base de soja (sem contar bebidas light, diet, zero, com adoçante ou bebidas com leite e iogurte). Os seis primeiros foram considerados alimentos marcadores de alimentação saudável (MAS); e os nove últimos, de alimentação não saudável (MANS). Essa divisão foi baseada não somente nas recomendações nutricionais para prevenção de doenças não transmissíveis, que levam, em conta, a densidade energética e a quantidade de gordura, sal, açúcar, fibras e micronutrientes nos alimentos 21, mas, também, em evidências que sugerem a associação dessas variáveis com fatores de risco para doenças crônicas não transmissíveis 21,22,23.

Nesse instrumento, a estrutura da pergunta foi: "Nos últimos 7 dias, em quantos dias você comeu (alimento)?”, e as opções de resposta foram: "não comi (alimento) nos últimos sete dias", "1 dia nos últimos sete dias", "2 dias nos últimos sete dias", "3 dias nos últimos sete dias”, "4 dias nos últimos sete dias”, "5 dias nos últimos sete dias”, "6 dias nos últimos sete dias" e "todos os dias nos últimos sete dias”.

As rotinas alimentares foram medidas por meio da frequência semanal (número de dias em que o aluno realizou determinada prática na semana que antecedeu o estudo) da presença de um responsável durante o almoço ou jantar (REFEIÇÃO), da realização do desjejum (DESJEJUM) e da prática de comer enquanto estuda ou assiste à TV. Essas rotinas foram escolhidas por terem se mostrado positivamente (no caso da não realização do desjejum e da prática de comer enquanto estuda ou assiste à TV) ou negativamente (no caso de realização de refeição com responsável) associadas ao excesso de peso e à obesidade 24,25,26.

\section{Recordatório de $\mathbf{2 4}$ horas (método} de referência)

Foram aplicados três recordatórios de 24 horas (R24h) por meio de entrevista telefônica realizada em dias alternados, sendo dois recordatórios referentes a dias de semana e um referente a um dia de final de semana. Foi criado um cronograma de ligações telefônicas que cobriu todos os dias da semana e que garantiu que todos eles fossem contemplados proporcionalmente no estudo. Caso não fosse possível falar com o adolescente na data da primeira tentativa de realização de algum dos três recordatórios, novo contato era feito na semana seguinte, no mesmo dia da semana, para execução do recordatório faltante.

Para o preenchimento do R24h, foi solicitado que o adolescente descrevesse espontaneamente o consumo de alimentos e bebidas no dia anterior à ligação desde a hora em que acordou até a hora em que foi dormir (recordatório espontâneo) 27. Em seguida, o pesquisador perguntou explicitamente sobre o consumo dos alimentos de interesse (incluídos no questionário da PeNSE) no dia anterior (recordatório simplificado) à ligação desde a hora em que acordou até a hora em que foi dormir 17,28,29. Adicionalmente, em cada entrevista, foi perguntado sobre a realização, no dia anterior, das atividades de interesse para os indicadores de rotinas alimentares (REFEIÇÃO, DESJEJUM e prática de comer enquanto estuda ou assiste à TV). Para o presente estudo, foram utilizados os dados dos R24h simplificados, conforme fundamentado adiante.

\section{Coleta de dados}

A primeira etapa do estudo consistiu na realização de visita às escolas para o preenchimento do questionário. Com o intuito de se reproduzirem as operações realizadas durante a coleta de dados da PeNSE, os adolescentes responderam ao questionário completo, ainda que o presente estudo tenha sido direcionado ao módulo de práticas alimentares. O preenchimento do questionário foi feito utilizando-se microcomputador de mão (personal digital assistant - PDA) da mesma versão que na edição de 2012 da PeNSE. Posteriormente, foram realizadas as ligações telefônicas para a realização dos R24h. A finalização dessa etapa ocorreu, no máximo, 15 dias após a aplicação do QUEST. A coleta de dados foi realizada de setembro a novembro de 2011 e durou 51 dias.

\section{Análise dos dados}

Com base na frequência alimentar obtida no questionário aplicado com PDA, foi construído o indicador que expressa a proporção de alunos que consomem regularmente (em pelo menos cinco dias da semana) cada um dos alimentos selecionados 2 . Procedimento similar foi realizado com as perguntas de rotina alimentar. Tendo em vista que a intenção do presente estudo foi 
validar os indicadores utilizados na PeNSE, o ponto de corte aqui adotado foi o mesmo daquele utilizado nas duas edições dessa pesquisa realizadas até o momento 9,10.

Em relação às perguntas de rotina alimentar, para extrapolar os dados obtidos por meio dos R24h em frequência semanal, os resultados obtidos nos recordatórios referentes a dias de semana foram multiplicados por 2,5, e os referentes ao dia de fim de semana, por 2,0. O somatório desses três valores foi tomado como a frequência semanal. Foram então construídos indicadores de ingestão e rotinas alimentares idênticos aos construídos com base no questionário aplicado com PDA.

O estudo de validade relativa se consistiu em comparar os indicadores de ingestão e rotinas alimentares obtidos por meio do QUEST (método teste) e dos R24h simplificados (método de referência).

A escolha de se utilizarem os dados dos recordatórios simplificados e não os do recordatório espontâneo foi baseada em dois conjuntos de informações. O primeiro refere-se a dois estudos que validaram esse tipo de instrumento $28,29 \mathrm{e}$ um estudo que o selecionou como método de referência para validar indicadores utilizados por sistema de vigilância específico para adolescente 17. O segundo conjunto advém de análises preliminares do nosso estudo. Comparando-se as proporções estimadas de indivíduos que consumiam regularmente ( $\geq 5$ dias na semana) cada um dos alimentos selecionados com base nos R24h espontâneos com aquelas obtidas com base nos R24h simplificados, observamos que, para quatro das seis perguntas referentes a alimentos MAS, elas foram maiores quando foram estimadas pela versão simplificada. Situação similar foi observada para os alimentos MANS: para seis dos nove alimentos estudados, as proporções foram mais altas quando foram estimadas pelos R24h simplificados. Os achados dessas análises preliminares corroboram os encontrados em outros estudos, que apontam que a abordagem explícita dos alimentos de interesse facilita o exercício de lembrança, por parte do entrevistado, do que ele consumiu no dia anterior 17,28, diminuindo, assim, a chance de viés de memória 27,30.

Com o objetivo de identificar se houve seletividade entre os adolescentes não avaliados, foram comparadas as variáveis idade, índice de massa corporal (IMC), escolaridade materna, sexo e dependência administrativa da escola da população avaliada e não avaliada aplicandose os testes Mann-Whitney (para idade), teste $t$ (para IMC) e qui-quadrado (para as outras três variáveis). Todas as análises foram realizadas para o total da amostra e, separadamente, por sexo. Calculou-se a proporção de adolescentes com ingestão e rotina alimentar regular ( $\geq 5$ dias/ semana) segundo dados do QUEST e do R24h. Utilizou-se o teste McNemar para comparar a diferença entre essas proporções, com nível de significância de 0,05.

Assumindo-se que a verdadeira condição dos indivíduos foi conhecida por meio dos R24h, para avaliar a capacidade do QUEST de classificálos corretamente segundo sua condição de ingestão e rotina alimentar (regular ou não), foram estimadas a sensibilidade (capacidade de classificar como positivo quem, de fato, apresenta a condição de interesse) e a especificidade (capacidade de classificar como negativo quem, de fato, não apresenta a condição de interesse) de cada indicador. Para sumarizar a concordância obtida para cada indicador, calculou-se o índice de acurácia do método teste, que é estimado por meio da proporção de classificações corretas (verdadeiros positivos e verdadeiros negativos) entre os indivíduos estudados 30 .

As análises estatísticas foram realizadas com o auxílio do SPSS versão 17 (SPSS Inc., Chicago, Estados Unidos) e do Microsoft Office Excel 2007 (Microsoft Corp., Estados Unidos).

\section{Aspectos éticos}

O estudo foi aprovado pelo Comitê de Ética em Pesquisa da Secretaria Municipal de Saúde e Defesa Civil do Rio de Janeiro, protocolo de pesquisa no 138/10. A participação dos alunos foi voluntária, podendo haver desistência em qualquer etapa do estudo. Assim como na PeNSE, o termo de consentimento livre e esclarecido de participação foi preenchido pelo próprio aluno na tela do PDA.

\section{Resultados}

Todas as escolas sorteadas aceitaram participar do estudo. Dos 251 alunos presentes nas nove turmas, foram efetivamente estudados 174 alunos (fluxograma de perdas na Figura 1). O principal motivo de não realizar a segunda etapa completa do estudo (três R24h) foi não conseguir contatar os alunos por telefone. Desses, $54 \%$ eram do sexo feminino, e $67,2 \%$ estudavam em escola pública. A faixa etária do grupo estudado variou de 13 a 17 anos, com média de idade de 14,7 anos. Houve diferença estatisticamente significativa ( $p=0,017)$ entre o grupo estudado e o não estudado em relação à dependência administrativa da escola, com maior proporção de adolescentes de escola privada entre os não estudados $(48,7 \%$ contra $32,8 \%)$. 
Não houve diferença na proporção de adolescentes com consumo regular de alimentos MAS estimada pelos dois métodos de aferição. Já dos nove alimentos MANS estudados, as estimativas geradas pelo QUEST foram mais altas para salgadinhos de pacote e biscoitos salgados e mais baixas para embutidos. Foi também observada menor frequência do indicador REFEIÇÃO quando foi utilizado o QUEST. Foram observados valores mais altos de especificidade que de sensibilidade para 12 dos 15 indicadores de ingestão alimentar. Já em relação aos três indicadores de rotinas alimentares, dois apresentaram valores mais altos de sensibilidade. Para o conjunto de indicadores, a especificidade variou de $53,1 \%$ a $92,5 \%$, e a sensibilidade, de $20,8 \%$ a $83,5 \%$. Todos os valores do índice de acurácia observados para os indicadores referentes a alimentos MAS, MANS e a rotinas alimentares variaram, respectivamente, de $71,8 \%$ a $83,3 \%, 59,8 \%$ a $88,4 \%$ e $61,5 \%$ a $82,2 \%$ (Tabela 1).

Analisando-se os dados segundo sexo, observou-se que, quando comparadas às estimativas de prevalência obtidas pelo R24h, as obtidas pelo
QUEST superestimaram o consumo regular de salgadinhos de pacote, biscoitos em geral, biscoitos salgados, biscoitos doces e guloseimas entre as meninas e subestimaram o consumo de embutidos e a prática de refeição com o responsável entre os meninos. Os resultados de maior especificidade para os indicadores de ingestão alimentar e de maior sensibilidade para os indicadores de rotina alimentar se mantiveram. Foram observados, ainda, entre os meninos, maiores índices de acurácia em 12 dos 18 indicadores estudados (Tabela 2).

\section{Discussão}

Os indicadores de ingestão alimentar e rotinas alimentares utilizados pela PeNSE apresentam alta acurácia. Em geral, a sensibilidade e a especificidade observadas para os indicadores referentes aos alimentos MAS e para o indicador DESJEJUM apresentaram melhor desempenho quando comparados aos MANS e aos demais indicadores de rotina alimentar. $\mathrm{O}$ mesmo foi encontrado

Figura 1

Fluxograma de participação no estudo de validação dos indicadores de práticas alimentares da Pesquisa Nacional de Saúde do Escolar (PeNSE). Escolares do 9o ano no Município do Rio de Janeiro, Brasil, 2011.

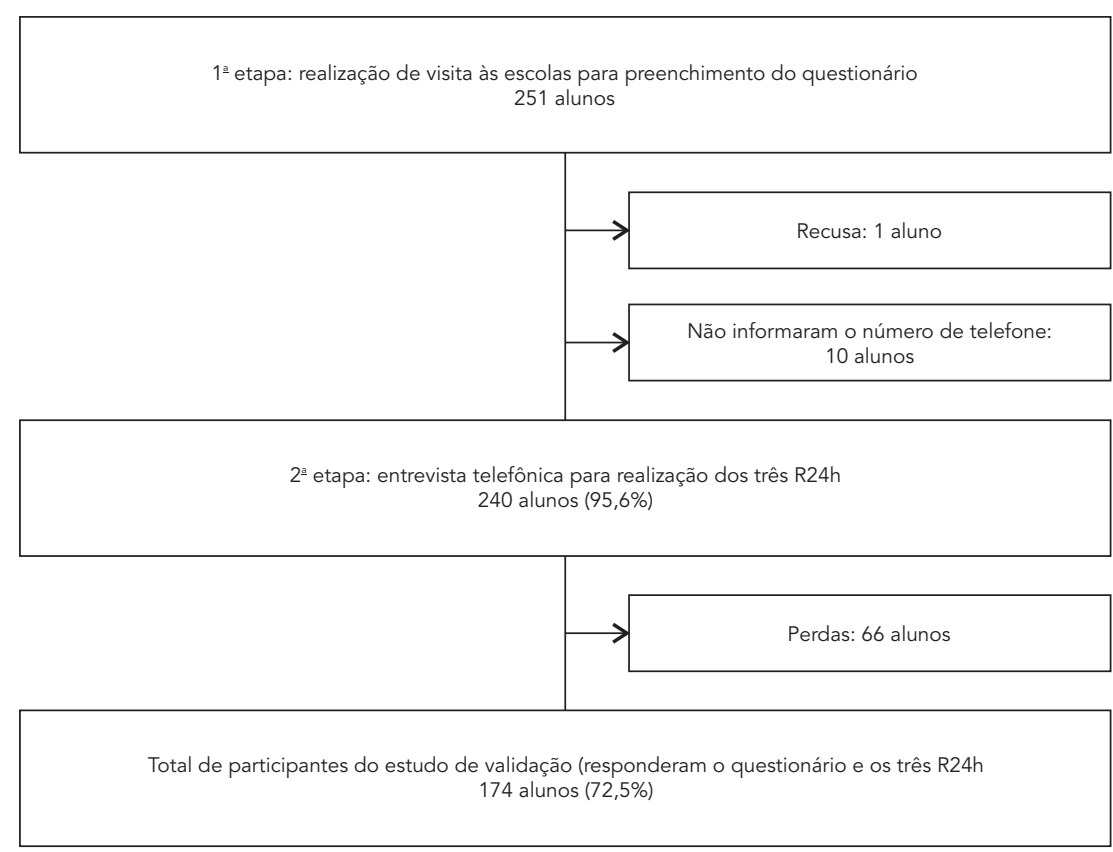

R24h: recordatórios de 24 horas. 
Tabela 1

Frequência (\%) de indicadores de consumo e prática alimentar regular ( $\geq 5$ dias/semana) segundo questionário de frequência alimentar (QUEST) e três recordatórios de 24 horas (R24h) e sensibilidade, especificidade e índice de acurácia do QUEST. Escolares do 9o ano no Município do Rio de Janeiro, Brasil, 2011.

\begin{tabular}{|c|c|c|c|c|c|}
\hline Variáveis & QUEST & R24h & $\begin{array}{l}\text { Sensibilidade do } \\
\text { QUEST (\%) }\end{array}$ & $\begin{array}{c}\text { Especificidade do } \\
\text { QUEST (\%) }\end{array}$ & $\begin{array}{c}\text { Índice de acurácia } \\
\text { do QUEST (\%) }\end{array}$ \\
\hline \multicolumn{6}{|l|}{ Ingestão alimentar } \\
\hline Hortaliça cozida & 14,9 & 12,1 & 42,9 & 88,9 & 83,3 \\
\hline Salada crua & 19,0 & 19,5 & 38,2 & 85,7 & 76,4 \\
\hline Fruta fresca & 31,0 & 24,1 & 57,1 & 77,3 & 74,1 \\
\hline Leite & 54,0 & 51,1 & 76,4 & 69,4 & 73,0 \\
\hline Hortaliça crua ou cozida & 30,5 & 29,3 & 54,9 & 79,7 & 72,4 \\
\hline Feijão & 67,2 & 67,8 & 78,8 & 57,1 & 71,8 \\
\hline Batata frita ou salgados fritos & 9,2 & 6,9 & 33,3 & 92,5 & 88,4 \\
\hline Salgadinho de pacote & $12,7^{*}$ & 4,6 * & 25,0 & 87,9 & 85,0 \\
\hline Biscoito salgado & 24,7 * & 10,8 * & 33,3 & 76,3 & 71,8 \\
\hline Refrigerante & 29,9 & 33,3 & 51,7 & 81,0 & 71,3 \\
\hline Embutido & 16,3 * & 28,2 * & 20,8 & 85,5 & 67,4 \\
\hline Biscoito em geral & 39,7 & 31,0 & 59,3 & 69,2 & 66,1 \\
\hline Biscoito doce & 28,2 & 19,0 & 30,3 & 72,3 & 64,4 \\
\hline Bebida açucarada & 55,2 & 62,6 & 63,3 & 58,5 & 61,5 \\
\hline Guloseima & 44,8 & 35,6 & 56,5 & 61,6 & 59,8 \\
\hline \multicolumn{6}{|l|}{ Rotina alimentar } \\
\hline Realizar desjejum & 64,4 & 69,5 & 83,5 & 79,2 & 82,2 \\
\hline Realizar refeição com responsável & 52,3 * & 63,8 * & 64,0 & 68,3 & 65,5 \\
\hline Comer assistindo à TV ou estudando & 58,6 & 53,4 & 68,8 & 53,1 & 61,5 \\
\hline
\end{tabular}

* Teste McNemar com valor de $\mathrm{p}<0,05$

Tabela 2

Frequência (\%) de indicadores de consumo e prática alimentar regular ( $\geq 5$ dias/semana) segundo questionário de frequência alimentar (QUEST) e três recordatórios de 24 horas (R24h) e sensibilidade, especificidade e índice de acurácia do QUEST segundo sexo. Escolares do 9o ano no Município do Rio de Janeiro, Brasil, 2011.

\begin{tabular}{|c|c|c|c|c|c|}
\hline Variáveis & QUEST & $\mathrm{R} 24 \mathrm{~h}$ & $\begin{array}{c}\text { Sensibilidade do } \\
\text { QUEST (\%) }\end{array}$ & $\begin{array}{c}\text { Especificidade do } \\
\text { QUEST (\%) }\end{array}$ & $\begin{array}{c}\text { Índice de acurácia do } \\
\text { QUEST (\%) }\end{array}$ \\
\hline \multicolumn{6}{|c|}{ Ingestão alimentar } \\
\hline \multicolumn{6}{|c|}{ Hortaliça cozida } \\
\hline Meninas & 17,0 & 10,6 & 40,0 & 85,7 & 80,9 \\
\hline Meninos & 12,5 & 13,8 & 45,5 & 92,2 & 86,3 \\
\hline \multicolumn{6}{|l|}{ Salada crua } \\
\hline Meninas & 22,3 & 19,1 & 38,9 & 81,6 & 73,4 \\
\hline Meninos & 15,0 & 20,0 & 37,5 & 90,6 & 80,0 \\
\hline \multicolumn{6}{|l|}{ Fruta fresca } \\
\hline Meninas & 28,7 & 25,5 & 50,0 & 78,6 & 71,3 \\
\hline Meninos & 33,8 & 22,5 & 66,7 & 75,8 & 73,8 \\
\hline \multicolumn{6}{|l|}{ Leite } \\
\hline Meninas & 54,3 & 48,9 & 80,4 & 70,8 & 75,5 \\
\hline Meninos & 53,8 & 53,8 & 72,1 & 67,6 & 70,0 \\
\hline
\end{tabular}

(continua) 
Tabela 2 (continuação)

\begin{tabular}{|c|c|c|c|c|c|}
\hline Variáveis & QUEST & $\mathrm{R}-24 \mathrm{~h}$ & $\begin{array}{l}\text { Sensibilidade do } \\
\text { QUEST (\%) }\end{array}$ & $\begin{array}{c}\text { Especificidade do } \\
\text { QUEST (\%) }\end{array}$ & $\begin{array}{c}\text { Índice de acurácia do } \\
\text { QUEST (\%) }\end{array}$ \\
\hline \multicolumn{6}{|c|}{ Ingestão alimentar } \\
\hline \multicolumn{6}{|c|}{ Hortaliça crua ou cozida } \\
\hline Meninas & 34,0 & 28,7 & 55,6 & 74,6 & 69,1 \\
\hline Meninos & 26,3 & 30,0 & 54,2 & 85,7 & 76,3 \\
\hline \multicolumn{6}{|l|}{ Feijão } \\
\hline Meninas & 66,0 & 60,6 & 80,7 & 56,8 & 71,3 \\
\hline Meninos & 68,8 & 76,3 & 77,0 & 57,9 & 72,5 \\
\hline \multicolumn{6}{|c|}{ Batata frita ou salgado frito } \\
\hline Meninas & 10,8 & 8,5 & 37,5 & 91,8 & 87,1 \\
\hline Meninos & 7,5 & 5,0 & 25,0 & 93,4 & 90,0 \\
\hline \multicolumn{6}{|c|}{ Salgadinho de pacote } \\
\hline Meninas & 13,8 * & 4,3 * & 50,0 & 87,8 & 86,2 \\
\hline Meninos & 11,4 & 5,0 & 0,0 & 88,0 & 83,5 \\
\hline \multicolumn{6}{|c|}{ Biscoito salgado } \\
\hline Meninas & 31,9 * & $8,5^{\star}$ & 50,0 & 69,8 & 68,1 \\
\hline Meninos & 16,3 & 12,5 & 20,0 & 84,3 & 76,3 \\
\hline \multicolumn{6}{|l|}{ Refrigerante } \\
\hline Meninas & 29,8 & 31,9 & 46,7 & 78,1 & 68,1 \\
\hline Meninos & 30,0 & 35,0 & 57,1 & 84,6 & 75,0 \\
\hline \multicolumn{6}{|l|}{ Embutido } \\
\hline Meninas & 20,2 & 26,6 & 28,0 & 82,6 & 68,1 \\
\hline Meninos & 11,5 * & 30,0 * & 13,0 & 89,1 & 66,7 \\
\hline \multicolumn{6}{|c|}{ Biscoito em geral } \\
\hline Meninas & 43,6 * & 28,7 * & 77,8 & 70,0 & 72,3 \\
\hline Meninos & 35,0 & 33,8 & 40,7 & 67,9 & 58,8 \\
\hline \multicolumn{6}{|c|}{ Biscoito doce } \\
\hline Meninas & 36,2 * & 17,0 * & 50,0 & 66,7 & 63,8 \\
\hline Meninos & 18,8 & 21,3 & 11,8 & 79,4 & 65,0 \\
\hline \multicolumn{6}{|c|}{ Bebida açucarada } \\
\hline Meninas & 57,4 & 63,8 & 65,0 & 55,9 & 61,7 \\
\hline Meninos & 52,5 & 61,3 & 61,2 & 61,3 & 61,3 \\
\hline \multicolumn{6}{|l|}{ Guloseima } \\
\hline Meninas & 57,4 * & 31,9 * & 66,7 & 46,9 & 53,2 \\
\hline Meninos & 30,0 & 40,0 & 46,9 & 81,2 & 67,5 \\
\hline \multicolumn{6}{|l|}{ Rotina alimentar } \\
\hline \multicolumn{6}{|c|}{ Realizar desjejum } \\
\hline Meninas & 59,6 & 64,9 & 80,3 & 78,8 & 79,8 \\
\hline Meninos & 70,0 & 75,0 & 86,7 & 80,0 & 95,0 \\
\hline \multicolumn{6}{|c|}{ Realizar refeição com responsável } \\
\hline Meninas & 55,3 & 58,5 & 70,9 & 66,7 & 69,1 \\
\hline Meninos & 48,8 * & 70,0 * & 57,1 & 70,8 & 61,3 \\
\hline \multicolumn{6}{|c|}{ Comer assistindo à TV ou estudando } \\
\hline Meninas & 56,4 & 51,1 & 64,6 & 52,2 & 58,5 \\
\hline Meninos & 61,3 & 56,3 & 73,3 & 54,3 & 65,0 \\
\hline
\end{tabular}

* Teste McNemar com valor de $p<0,05$. 
para o índice de acurácia e para a comparação das estimativas de frequência.

Quando avaliado segundo sexo, os indicadores apresentaram maior acurácia entre os meninos. Embora a validade dos indicadores seja satisfatória para as meninas, o desempenho do instrumento da PeNSE é melhor para os meninos.

Os valores de especificidade superiores aos de sensibilidade para os indicadores de ingestão alimentar (tanto MAS quanto MANS) indicam que eles são capazes de identificar melhor os adolescentes que não ingerem regularmente os alimentos avaliados, ou seja, que não estão expostos aos fatores de risco e proteção examinados na PeNSE.

Entre os alimentos MANS, a frequência estimada pelo QUEST para os indicadores referentes à ingestão regular de bebidas açucaradas e refrigerantes foi similar à estimada pelos R24h. Por outro lado, foi observada superestimação do consumo regular de biscoitos salgados e de salgadinhos de pacote e subestimação do consumo regular de embutidos. A interpretação da diferença dessas estimativas não é tarefa simples. Em geral, questionários de frequência alimentar tendem a superestimar a ingestão ${ }^{27}$. Uma possibilidade de explicação seria o fato de o agrupamento de uma gama de itens em uma mesma questão ter dificultado o entendimento e comprometido a resposta 11,27. Exemplo disso seria a pergunta referente ao consumo de embutidos, na qual foram listados oito alimentos. Outro motivo poderia ser a dificuldade de o aluno informar a ingestão de determinados alimentos em função de consumi-los frequentemente enquanto realiza outra atividade, como, por exemplo, enquanto assiste à TV ou joga videogame 31,32 . Um terceiro motivo poderia ser a dificuldade de diferenciar os itens contemplados na questão referente a biscoitos salgados daqueles abarcados na questão sobre salgadinhos de pacote por não saberem a diferença entre os dois termos. Uma possível solução para isso seria a inclusão de exemplos desses alimentos como já é feita em outras questões.

A comparação dos nossos achados com os dos demais estudos de validação referentes aos sistemas de vigilância dirigidos a adolescentes existentes no mundo é limitada em função das diferenças nas características da população, nos questionários e indicadores utilizados, no método de referência adotado e na análise estatística empregada. Ainda assim, pode-se observar que frequências de consumo de alimentos MAS e MANS estimadas pelo método teste (questionários dos sistemas de vigilância) e pelo método de referência também apresentaram diferenças em outras realidades.
No presente estudo, não foi observada diferença estatisticamente significativa entre as proporções de ingestão regular de cada um dos alimentos MAS estimadas pelos dois métodos. O mesmo desempenho não foi observado pelos estudos de validação que avaliaram os indicadores gerados com base nos questionários do Health Behaviour in School-aged Children (HBSC) e do Youth Risk Behavior Surveillance System (YRBSS). Em relação ao consumo de frutas e hortaliças, foi observada superestimação pelo questionário do HBSC e subestimação pelo YRBSS 16,17,18. O questionário do HBSC também superestimou o consumo de leite com redução do teor de gordura para adolescentes belgas e italianos. Em relação ao consumo de leite integral, houve superestimação para os belgas e subestimação para os italianos 18 .

Em relação aos alimentos MANS, o consumo de guloseimas foi superestimado tanto pelo indicador utilizado na PeNSE (para o sexo feminino) quanto pelo adotado no HBSC (que não apresenta estratificação por sexo) ${ }^{18}$. O consumo regular de refrigerante foi adequadamente estimado na PeNSE e no HBSC, quando esse foi realizado com adolescentes belgas, e subestimado, quando foi aplicado a adolescentes italianos 18 .

A comparação do desempenho dos instrumentos nacional e europeu em relação à ingestão de salgadinhos de pacote deve ser feita com cautela, uma vez que, no Brasil, o questionário abarca um amplo leque de produtos, enquanto o HBSC contempla somente batata frita industrializada. O mesmo se aplica aos salgados fritos: em nosso país, consideramos diversas preparações e, no HBSC, é considerada somente a batata frita artesanal. Feita essa ressalva, observa-se que a ingestão regular de salgadinhos de pacote é superestimada tanto na PeNSE quanto no HBSC, e que a de salgados fritos é adequadamente estimada no Brasil, subestimada na Bélgica e superestimada na Itália 18 .

Dos 18 indicadores avaliados nesse estudo, 11 apresentaram índice de acurácia superiores a $70 \%$. Dado que esse índice permite observar o desempenho dos indicadores de maneira global 30 , ele parece ser um bom parâmetro para avaliar o desempenho de indicadores no contexto de sistemas de vigilância, uma vez que esses pretendem avaliar os fatores de risco e proteção à saúde no âmbito coletivo. Não foi encontrada nenhuma publicação referente a estudos de avaliação dos instrumentos utilizados em sistemas de vigilância que tenha incluído esse índice no elenco de parâmetros examinados. Por consequência, não foi possível o cotejamento de nossos achados com os de outros sistemas de vigilância. 
Comparando este estudo com outro que teve por objetivo desenvolver uma medida de triagem para o consumo diário de, pelo menos, cinco porções de frutas e hortaliças entre adolescentes 33 , observamos que esse apresentou resultados semelhantes aos nossos: índice de acurácia de $63 \%$ e especificidade maior que sensibilidade (63\% e 33\% respectivamente). Já outro estudo de validação de um instrumento de avaliação de ingestão de gordura realizado com adolescentes da Califórnia apresentou acurácia similar à observada para muitos dos indicadores aqui estudados (71\%), mas o valor de sensibilidade (81\%) foi maior que o de especificidade (47\%) 34 .

Não foi possível comparar nossos resultados referentes à maior acurácia dos indicadores entre meninos com os de outros estudos de validação de indicadores de sistemas de vigilância dirigidos a adolescentes, uma vez que esses não apresentaram análises segundo sexo 16,17,18. Também os estudos de validação de questionários de consumo alimentar voltados para adolescentes, em sua maioria, não analisam os dados segundo sexo 35,36. Entretanto, em um estudo de validação de um questionário de frequência alimentar, conduzido na Austrália com 785 adolescentes participantes de uma coorte de base populacional, foram observados resultados semelhantes aos nossos: maior superestimação do consumo entre meninas e melhor desempenho geral do instrumento entre meninos 37 .

No tocante aos aspectos metodológicos, cabe comentar algumas limitações já registradas na literatura em relação aos estudos de validação referentes a indicadores de ingestão alimentar, quais sejam: a definição do método utilizado como referência ou padrão ouro, a forma de aplicação dos métodos teste e de referência, a sequência de aplicação dos instrumentos e a extrapolação dos resultados para população que fará uso do instrumento $11,27,30,38,39$.

Não existe nenhum método de aferição absolutamente preciso para ser utilizado como referência em estudos de validação da ingestão alimentar. Os dois métodos mais utilizados como referência são as médias de consumo calculadas com base em diversas aplicações de registro alimentar e o R24h 27,39. A utilização do registro alimentar como referência pressupõe maior cooperação do indivíduo estudado 27 e pode acarretar sub-registro (não relatar alimentos e/ou refeições que foram consumidos) e/ou subconsumo (real diminuição do consumo por ter que preencher o instrumento) ${ }^{39}$. O R24h, por sua vez, depende da memória recente do entrevistado. Por outro lado, exige menor participação e motivação do entrevistado 39. O R24h foi utilizado como método de referência no presente estudo de validade relativa assim como nos trabalhos realizados para avaliar os questionários utilizados por outros sistemas de vigilância como o VIGITEL (Vigilância de Fatores de Risco e Proteção para Doenças Crônicas por Inquérito Telefônico) 38,40,41, YRBSS 16 e HBSC 17. Uma potencial limitação do uso de R24h em estudos de validação é a possibilidade de o dia investigado ser atípico, isto é, não expressar adequadamente o hábito alimentar do indivíduo estudado 27 . Para minimizar essa limitação, foram realizados três R24h em dias alternados, incluindo um dia de fim de semana.

Outra potencial limitação a ser considerada foi a utilização de uma versão simplificada do R24h. Embora sua aplicação facilite a lembrança do entrevistado 17,29, contribuindo para redução do viés de memória 42 e venha sendo adotada em estudos de validação como método de referência 17,29 , ela traz potencialmente a chance de viés de informação, seja, por exemplo, via superestimação do consumo de alimentos MAS ou subestimação dos alimentos MANS 42. Em tese, esse mesmo erro pode ocorrer quando se utiliza o método teste aqui adotado (QUEST). Isso não seria desejável em um estudo de validação, uma vez que estariam sendo comparados dois instrumentos sujeitos aos mesmos erros, podendo levar a resultados mais satisfatórios do que realmente seriam $27,28,42$.

Entretanto, conforme já mencionado na seção de metodologia, nas análises preliminares de nosso estudo, observamos que as proporções de consumo regular foram, em sua maioria, superiores, quando foram estimadas pelo R24h, se comparadas às estimadas pelos recordatórios espontâneos, tanto para alimentos MAS quanto MANS. Esse achado sugere que a abordagem explícita dos alimentos de interesse não parece ter introduzido viés de informação. Resultado semelhante foi observado por Kristal et al. ${ }^{28}$, tanto o consumo de alimentos desejáveis quanto o de indesejáveis foram relatados com mais frequência no R24h que no recordatório espontâneo.

Uma vantagem do método de referência adotado é o fato de o R24h ter sido aplicado por telefone, o que permitiu representar igualmente os dias da semana, uma vez que foram realizadas entrevistas aos sábados e domingos para aplicar os R24h referentes aos dias de sexta-feira e sábado, respectivamente. Esse é um diferencial do estudo, uma vez que, comumente, em pesquisas dirigidas a escolares, a coleta de dados é realizada na própria escola, entre segunda e sexta-feira. Outra vantagem diz respeito ao fato de, na aplicação do R24h, ter sido perguntado explicitamente sobre os alimentos de interesse, o que facilitou a lembrança, pelos adolescentes, dos alimentos ingeridos na véspera 17,29,38. Além disso, a aplicação 
do R24h por telefone é cada vez mais utilizada e apresenta custo menor do que o das entrevistas face a face 42 .

Uma potencial fragilidade do estudo é o fato de o método teste ter sido aplicado na forma de questionário autopreenchido, e o método de referência ter sido realizado por meio de entrevistas telefônicas. Uma revisão sobre inquéritos por telefone como método para obtenção de informações dietéticas destaca que as entrevistas telefônicas produzem resultados que são comparáveis aos obtidos por outros métodos de aplicação, e que os estudos de validação apresentam resultados positivos 43. Estudos de validação que compararam o desempenho de $\mathrm{R} 24 \mathrm{~h}$ realizados por telefone e presencialmente indicam que a entrevista telefônica é um método válido para avaliar o consumo alimentar 44,45.

Em relação à sequência de aplicação dos instrumentos, nos estudos de validação, o método testado deve ser administrado antes do método de referência, de forma a evitar que o preenchimento desse último influencie o resultado do primeiro ${ }^{39}$. Por esse motivo, o presente estudo comparou duas semanas distintas. Portanto, parte da diferença encontrada entre os dois métodos pode ser explicada pela variação real na ingestão dos alimentos estudados e nas rotinas alimentares dos adolescentes. Com objetivo de minimizar essa diferença, os três R24h foram aplicados no menor intervalo de tempo possível após o preenchimento do QUEST no PDA.

No tocante à extrapolação dos resultados encontrados, idealmente os indivíduos incluídos em um estudo de validação devem apresentar variabilidade das medidas de interesse semelhante à da população para qual o instrumento será aplicado 11,27. No caso de um grupo não representado na amostra, um instrumento pode ser considerado válido para um grupo populacional com características similares àquelas do grupo estudado. Se as circunstâncias forem outras, por exemplo, diferente nível educacional ou socioeconômico, o resultado do estudo ori- ginal de validação pode não ser aplicado, e novos estudos serão necessários 11,27. Os achados aqui relatados não refletem, necessariamente, o real desempenho do questionário de consumo alimentar e práticas alimentares utilizado pelo sistema de vigilância no restante do país. Entretanto, é razoável sugerir que os resultados sejam semelhantes nas capitais com perfil sociodemográfico semelhante ao do Rio de Janeiro. Ressalta-se, também, que a precisão das estimativas segundo sexo dos adolescentes é menor do que as estimativas para o conjunto dos alunos, dado o tamanho da população efetivamente estudada em cada categoria de sexo.

Cabe ainda comentar que, no grupo estudado, a proporção de alunos inseridos em escolas particulares $(32,8 \%)$ foi superior à observada na PeNSE de 2009 para o Rio de Janeiro e para o conjunto de capitais brasileiras e Distrito Federal (23,4\% e $20,8 \%$, respectivamente). Isso pode ter ocorrido pelo fato de o número de alunos existentes nas escolas particulares ser maior, e o número daqueles nas escolas públicas ser menor que o previsto para o município no Censo Escolar de 2010. Mesmo havendo mais perdas entre alunos de escolas particulares, esse estrato ficou sobrerrepresentado na amostra estudada. Para superar parcialmente essa fragilidade, as análises foram realizadas por dependência administrativa da escola. Para o total de alunos estudados, entretanto, os resultados encontrados para a performance dos indicadores podem estar superestimados.

Os resultados do presente estudo indicam que os indicadores de consumo regular de alimentos MAS e aquele referente à realização do desjejum apresentaram melhor desempenho do que aqueles de consumo regular de alimentos MANS e os outros dois indicadores de rotinas alimentares. O conhecimento mais seguro sobre a validade dos indicadores de ingestão e rotinas alimentares gerados pelo instrumento utilizado na PeNSE requer a realização de estudos similares em outras realidades do país. 


\section{Resumen}

Se evaluó la validez relativa de los indicadores de prácticas alimentarias del cuestionario utilizado en la Investigación Nacional de Salud Escolar (PeNSE) entre adolescentes en la ciudad de Río de Janeiro, Brasil. Se estudiaron a 174 estudiantes. Se evaluaron los indicadores: consumo regular ( $\geq 5$ veces/semana) de los alimentos marcadores de alimentación saludable, marcadores de alimentación no saludable y las rutinas de alimentación (hacer las comidas con los responsables (COMIDA), el desayuno (DESAYUNO) y comer mientras estudian o ven la televisión. Se compararon los resultados del cuestionario y los tres recordatorios de 24 horas. Para todos los marcadores de alimentación saludable no hubo diferencia en la proporción del consumo regular, de acuerdo con ambos métodos. Las frecuencias generadas por el cuestionario fueron más altas para los indicadores snack pack $y$ galletas saladas e inferior en COMIDA. Los indicadores de consumo regular de marcadores de alimentación saludable y que se refieren al DESAYUNO eran aquellos con mayor precisión. Los indicadores de prácticas alimentarias utilizados por la PeNSE mostraron una validación satisfactoria.

Consumo de Alimentos; Hábitos Aliemnticios; Estudios de Validación; Adolescente

\section{Colaboradores}

L. F. Tavares e I. R. R. Castro contribuíram no delineamento do estudo, na supervisão do trabalho de campo, na análise do banco de dados e na redação do manuscrito. R. B. Levy e L. O. Cardoso colaboraram no delineamento do estudo e na revisão do manuscrito. M. D. Passos participou no delineamento do estudo, na supervisão do trabalho de campo e na revisão do manuscrito. F. S. B. Brito contribuiu na análise do banco de dados e na revisão do manuscrito.

\section{Agradecimentos}

Ao Instituto de Nutrição Annes Dias pelo apoio logístico para realização do trabalho de campo. Ao Instituto Brasileiro de Geografia e Estatística (IBGE) pelo empréstimo dos personal digital assistant (PDA). À FAPERJ (processo E-26/110.635/2011) e ao CNPq (processo 475782/2011-9) pelo financiamento.

\section{Referências}

1. Ministério da Saúde. A vigilância, o controle e a prevenção das doenças crônicas não transmissíveis: DCNT no contexto do Sistema Único de Saúde brasileiro. Brasília: Ministério da Saúde; 2005.

2. Castro IRR, Cardoso LO, Egstrom EM, Levy RB, Monteiro CA. Vigilância de fatores de risco para doenças não transmissíveis entre adolescentes: a experiência da cidade do Rio de Janeiro, Brasil. Cad Saúde Pública 2008; 24:2279-88.

3. World Health Organization; United Nations Children's Fund. Measurement of adolescent development: environmental, contextual and protective factors. Geneva: World Health Organization; 1999.

4. Brener ND, Kann L, Kinchen SA, Grunbaum JA, Whalen L, Eaton D, et al. Methodology of the youth risk behavior surveillance system. MMWR Recomm Rep 2004; 53(RR-12):1-13.
5. Currie C, Roberts C, Morgan A, Smith R, Settertobulte W, Samdal O, et al. Young people's health in context: Health Behaviour in School-aged Children (HBSC) study: international report from the 2001/2002 survey. Geneva: World Health Organization; 2004.

6. Division of Adolescent and School Health, Centers for Disease Control and Prevention. Global School-based Student Health Survey (GSHS) 2009. Atlanta: Centers for Disease Control and Prevention; 2009.

7. Instituto Nacional de Câncer. VIGESCOLA: vigilância de tabagismo em escolares. Dados e fatos de 12 capitais brasileiras. v. 1. Rio de Janeiro: Instituto Nacional de Câncer; 2004. 
8. Monteiro CA, Moura EC, Jaime PC, Lucca A, Florindo AA, Figueiredo ICR, et al. Monitoramento de fatores de risco para doenças crônicas por entrevistas telefônicas. Rev Saúde Pública 2005; 39 : 47-57.

9. Instituto Brasileiro de Geografia e Estatística. Pesquisa Nacional de Saúde Escolar 2009. Rio de Janeiro: Instituto Brasileiro de Geografia e Estatística; 2010.

10. Instituto Brasileiro de Geografia e Estatística. Pesquisa Nacional de Saúde Escolar 2012. Rio de Janeiro: Instituto Brasileiro de Geografia e Estatística; 2013.

11. Streiner DL, Norman GR. Health measurement scales: a practical guide to their development and use. $4^{\text {th }}$ Ed. New York: Oxford University Press; 2008.

12. Becker AE, Thomas JJ, Bainivualiku A, Richards L, Navara K, Roberts AL, et al. Validity and reliability of a Fijian translation and adaptation of the Eating Disorder Examination Questionnaire. Int J Eat Disord 2010; 43:171-8.

13. Booth ML, Okely AD, Bauman A. The reliability and validity of the physical activity questions in the WHO health behaviour in schoolchildren (HBSC) survey: a population study. Br J Sports Med 2001; 35:263-7.

14. Troped PJ, Fragala MS, Finkelstein DM, Kim J, Peterson KE. Reliability and validity of YRBS physical activity items among middle school students. Med Sci Sports Exerc 2007; 39:416-25.

15. Rangul V, Holmen TL, Kurtze N, Cuypers K, Midthjell K. Reliability and validity of two frequently used self-administered physical activity questionnaires in adolescents. BMC Med Res Methodol 2008; 8:47.

16. Field AE, Colditz GA, Fox MK, Byers T, Serdula M, Bosch RJ, et al. Comparison of 4 questionnaires for assessment of fruit and vegetable intake. Am J Public Health 1998; 88:1216-8.

17. Vereecken C, Maes LA. A Belgian study on the reliability and relative validity of the Health Behaviour in School-Aged Children Food Frequency Questionnaire. Public Health Nutr 2003; 6:581-8.

18. Vereecken CA, Rossi S, Giacchi MV, Maes L. Comparison of a short food-frequency questionnaire and derived indices with a seven-day diet record in Belgian and Italian children. Int J Public Health 2008; 53:297-305.

19. Cardoso LO. Fatores associados ao excesso de peso e perfis de consumo e comportamento alimentar de adolescentes [Tese de Doutorado]. Rio de Janeiro: Escola Nacional de Saúde Pública Sergio Arouca, Fundação Oswaldo Cruz; 2010.

20. Reichenheim ME. Sample size for the kappa-statistic of interrater agreement. Stata Technical Bulletin 2000; 58:41-5.

21. World Health Organization. Diet, nutrition and the prevention of chronic diseases. Geneva: World Health Organization; 2003.
22. Agudo A, Cabrera L, Amiano P, Ardanaz E, Barricarte A, Berenguer T, et al. Fruit and vegetable intakes, dietary antioxidant nutrients, and total mortality in Spanish adults: findings from the Spanish cohort of the European Prospective Investigation into Cancer and Nutrition (EPIC-Spain). Am J Clin Nutr 2007; 85:1634-42.

23. Dauchet L, Amouyel P, Hercberg S, Dallongeville J. Fruit and vegetable consumption and risk of coronary heart disease: a meta-analysis of cohort studies. J Nutr 2006; 136:2588-93.

24. Videon TM, Manning CK. Influences on adolescent eating patterns: the importance of family meals. J Adolesc Health 2003; 32:365-73.

25. Patrick H, Nicklas TA. A review of family and social determinants of children's eating patterns and diet quality. J Am Coll Nutr 2005; 24:83-92.

26. Fitzpatrick E, Edmunds LS, Dennison BA. Positive effects of family dinner are undone by television viewing. J Am Diet Assoc 2007; 107:666-71.

27. Willet W. Nutritional epidemiology. 2nd Ed. New York: Oxford University Press; 1998.

28. Kristal AR, Abrams BF, Thornquist MD, Disogra L, Croyle RT, Shattuck AL, et al. Development and validation of a food use checklist for evaluation of community nutrition interventions. Am J Public Health 1990; 80:1318-22.

29. Smith KW, Hoelscher DM, Lytle LA, Dwyer JT, Nicklas TA, Zive MM, et al. Reliability and validity of the Child and Adolescent Trial for Cardiovascular Health (CATCH) Food Checklist: a self-report instrument to measure fat and sodium intake by middle school students. J Am Diet Assoc 2001; 101:635-47.

30. Szklo M, Javier Nieto F. Epidemiology: beyond the basics. 2nd Ed. Maryland: Aspen; 2007.

31. Moray J, Fu A, Brill K, Mayoral MS. Viewing television while eating impairs the ability to accurately estimate total amount of food consumed. Bariatr Nurs Surg Patient Care 2007; 2:71-6.

32. Oldham-Cooper RE, Hardman CA, Nicoll CE, Rogers PJ, Brunstrom JM. Playing a computer game during lunch affects fullness, memory for lunch, and later snack intake. Am J Clin Nutr 2011; 93:308-13.

33. Prochaska JJ, Salles JF. Reliability and validity of a fruit and vegetable screening measure for adolescents. J Adolesc Health 2004; 34:163-5.

34. Prochaska JJ, Sallis JF, Rupp J. Screening measure for assessing dietary fat intake among adolescents. Prev Med 2001; 33:699-706.

35. Slater B, Philippi ST, Fisberg RM, Latorre MRDO. Validation of a semi- quantitative adolescent food frequency questionnaire applied at a public school in São Paulo, Brazil. Eur J Clin Nutr 2003; 57: 629-35.

36. Araujo MC, Yokoo EM, Pereira RA. Validation and calibration of a semiquantitative food frequency questionnaire designed for adolescents. J Am Diet Assoc 2010; 110:1170-7. 
37. Ambrosini GL, Klerk NH, O’Sullivan TA, Beilin LJ, Oddy $\mathrm{WH}$. The reliability of a food frequency questionnaire for use among adolescents. Eur J Clin Nutr 2009; 63:1251-9.

38. Monteiro CA, Moura EC, Jaime PC, Claro RM. Validade de indicadores do consumo de alimentos e bebidas obtidos por inquérito telefônico. Rev Saúde Pública 2008; 42:582-9.

39. Slater B, Philippi ST, Marchioni DML, Fisberg RM. Validação de Questionários de Frequência Alimentar - QFA: considerações metodológicas. Rev Bras Epidemiol 2003; 6:200-8.

40. Neves ACM, Gonzaga LAA, Martens IBG, Moura EC. Validação de indicadores do consumo de alimentos e bebidas obtidos por inquérito telefônico em Belém, Pará, Brasil. Cad Saúde Pública 2010; 26:2379-88.

41. Mendes LL, Campos SF, Malta DC, Bernal RTI, Sá NNB, Velásquez-Meléndez G. Validade e reprodutibilidade de marcadores do consumo de alimentos e bebidas de um inquérito telefônico realizado na cidade de Belo Horizonte (MG), Brasil. Rev Bras Epidemiol 2011; 14 Suppl 1:80-9.
42. Gibson RS. Principles of nutritional assessment. 2nd Ed. New York: Oxford University Press; 2005.

43. Fox TA, Heimendinger J, Block G. Telephone surveys as a method for obtaining dietary information: a review. J Am Diet Assoc 1992; 92:729-32.

44. Casey PH, Goolsby SLP, Lensing SY, Perloff BP, Bogle ML. The use of telephone interview methodology to obtain 24-hour dietary recalls. J Am Diet Assoc 1999; 99:1406-11.

45. Tran KM, Johnson RK, Soultanakis RP, Matthews DE. In-person vs. telephone-administered multiple-pass 24-hour recalls in women: validation with doubly labeled water. J Am Diet Assoc 2000; 100:777-83.

Recebido em 03/Jan/2013

Versão final reapresentada em 24/Set/2013

Aprovado em 04/Nov/2013 